\title{
Uso e importancia de los recursos naturales y su incidencia en el desarrollo turístico. Caso Cantón Chilla, El Oro, Ecuador
}

\section{Use and importance of the natural resources and their impact on tourism development. Case of Chilla Canton, El Oro, Ecuador}

Jordy Alexander Orellana Salas ${ }^{1}$, Tatiana Del Cisne Lalvay Portilla ${ }^{2}$

Resumen: El crecimiento turístico en un determinado lugar está estrechamente sujeto a la forma en como sus habitantes hacen uso de los recursos naturales, la importancia que estos tienen en su calidad de vida y subsistencia. El presente artículo tiene como objetivo realizar un análisis del uso e importancia de los recursos naturales y su incidencia en el desarrollo turístico del cantón Chilla, provincia El Oro, Ecuador. La estrategia metodológica fue la observación directa, se aplicó una encuesta a la población económicamente activa. Los resultados permitieron identificar los recursos naturales de mayor uso e importancia para la comunidad. Se propone la promoción de su conservación para mejorar la calidad de vida de los habitantes y lograr un turismo sustentable en el área. Ello porque una de las fuentes de ingresos económicos de la población es el desarrollo del turismo local, el cual debe ser realizado considerando la vulnerabilidad de los ecosistemas y respetando y cuidando el entorno natural. El cantón Chilla tiene una gran afluencia de turistas cada año por lo que es necesario concienciar a la población en el cuidado y buen manejo de los recursos naturales que poseen.

Palabras clave: Crecimiento turístico, recursos naturales, conservación, turismo local.
Abstract: Tourist growth in a certain place is closely contingent upon how its inhabitants make use of the natural resources and the importance these resources have in their quality of life and livelihood. This article seeks to analyze the use and importance of the natural resources and their impact on tourism development in Chilla Canton, El Oro Province, Ecuador. The methodological strategy was direct observation; a survey was administered to the economically active population. The results identified the natural resources of greatest use and importance to the community. Promoting their conservation is proposed to improve the inhabitants' quality of life and to achieve viable tourism in the area. This is because one of the population's sources of income is local tourism, which must be undertaken with an awareness of the vulnerability of ecosystems and respecting and taking care of the natural surroundings. Chilla Canton has a large influx of tourists every year, which is why the population must be mindful of the care and good management of the natural resources they possess.

Key words: Tourism growth, natural resources, conservation, local tourism.

(Presentado: 2 de septiembre de 2017. Aceptado: 28 de octubre de 2017)

\footnotetext{
${ }^{1}$ Administración de Hotelería y Turismo, Universidad Técnica de Machala, Ecuador. E-mail: jordy.orellanabscAggmail.com

${ }^{2}$ Administración de Hotelería y Turismo, Universidad Técnica de Machala, Ecuador. E-mail: tatianita2311 Qgmail.com
} 


\section{INTRODUCCIÓN}

El Cantón Chilla, con una extensión de 389 km2, forma parte de los 14 cantones que conforman la provincia de $\mathrm{El}$ Oro en Ecuador. Se encuentra ubicado en el occidente de un ramal montañoso de la cordillera de Los Andes con una altitud que va desde los 600 hasta los 3.813 msnm.

Actualmente, los recursos naturales son aprovechados por el ser humano para satisfacer sus necesidades de subsistencia, tales como alimentación, salud, económicas y de ocio; éstos se han convertido en una fuente de vida y desarrollo para la comunidad que habita en este lugar. En el momento que el hombre hace uso de estos recursos no solo obtiene beneficios personales, sino también ayuda a que la comunidad tenga un mejor desarrollo local y turístico. Todo aprovechamiento de recursos naturales deberá estar sujeto a los tres ejes de la sostenibilidad, siendo estos ambientales, sociales y económicos, que manteniendo así un comportamiento amigable con el medio ambiente, y sin comprometer el uso de los mismos recursos a las futuras generaciones. Los recursos naturales pueden claramente ser considerados como importantes generadores de ingresos para una población, pero el poco conocimiento de las personas acerca de la riqueza de los mismos, conlleva a un escaso nivel de valoración, deficiente manejo para su utilización, por lo que genera un impacto negativo al medio ambiente y al desarrollo del lugar. Haciendo énfasis a lo que dicen Quizhpe et al. (2017) lamentablemente en Ecuador, un país considerado megadiverso y con importantes áreas naturales, son escasos y limitados los estudios e investigaciones que se han llevado a cabo con la finalidad de conocer el grado de importancia de los recursos naturales para las personas que habitan en estas áreas y hacen uso de los mismos.

Actualmente, la comunidad del cantón Chilla no posee los conocimientos necesarios sobre el correcto uso de los recursos naturales que tiene; por consiguiente, es un problema que afecta no solo a las personas que ahí habitan, sino a todo su ecosistema, ocasionando su deterioro e inclusive amenazando con la posibilidad de extinción de especies de flora y fauna nativa del lugar. Para esta investigación se realizaron visitas in situ, aplicando herramientas de investigación como la observación directa, investigación bibliográfica y aplicación de encuestas, siendo la muestra la población económicamente activa del cantón, cuyo objetivo fue realizar de los recursos naturales y su incidencia en el desarrollo turístico del cantón Chilla. De esta manera la población y entes competentes pueden hacer uso de esta información y ayudar en futuros proyectos encaminados a controlar la degradación de los recursos naturales que están siendo aprovechados sin conocimiento alguno de sostenibilidad, ya sea para actividades turísticas o personales.

La Organización Mundial de Turismo (OMT, 2013) indica que el turismo comprende las actividades que realizan las personas durante sus viajes y estancias en lugares distintos al de su entorno habitual, por un período de tiempo consecutivo inferior a un año con fines de ocio, por negocios y otros. En la actualidad el turismo es una de las actividades más importantes en el mundo y cada vez toma mayor relevo en el comercio internacional. La OMT (2013) hace énfasis en que el desarrollo del turismo puede traer beneficios para los países en desarrollo, y un tipo de turismo esencial en los que se puede trabajar es el turismo sostenible. En Ecuador el turismo ocupa un puesto importante, constituyéndose en la tercera fuente de ingresos para el país. Además, se ha convertido en pieza fundamental para el mejoramiento de la calidad de vida de la población. De acuerdo a Ferrán et al. (2016), es por estas razones que el Estado ecuatoriano creó la marca país "Ecuador ama la vida", impulsando una campaña de promoción turística no solo local sino internacional, con el objetivo de dar a conocer al resto del mundo sus riquezas naturales, socioculturales, religiosas y de biodiversidad, creando así una nueva perspectiva para nuevos proyectos turísticos, buscando inversionistas que reactiven este sector tan importante para la economía del país.

La sostenibilidad es una acción que permite a un lugar desarrollarse en diferentes ámbitos, como ambiental, económico y social, teniendo en cuenta que no afecte en el futuro las necesidades de la sociedad, ya que esto es un factor importante para que un lugar o destino crezca de manera sostenible. Esto implica crear mayor armonía entre naturaleza y sociedad (Bertoni, 2005). Hoy en día nadie pone en duda que la actividad turística debe ser una práctica sostenible que contribuya al desarrollo económico, la equidad social, la revalorización cultural y la preservación del entorno (Delgado, 2013). Cada vez más la contaminación en el planeta abarca un tema importante de tratar, esto se debe al agotamiento de los recursos naturales y al 
calentamiento global que afecta a toda la humanidad de diferentes maneras. Vargas (2016) aclara que la sostenibilidad juega un papel fundamental que busca no poner en peligro al medio ambiente ni los recursos naturales que existen en el lugar. Por su parte, Hernanz \& Gil (2013) expresan que actualmente el Estado ecuatoriano se encuentra sujeto a una nueva forma de desarrollo sociopolítico, cuyo enfoque principal ha sido la sostenibilidad, en este sentido se ha considerado al turismo sostenible y su desarrollo en un lugar preponderante dentro de la nueva Constitución de la República en donde existen leyes como la Ley de Aguas, Ley de Prevención y Control de la Contaminación Ambiental y sus reglamentos, Ley Forestal y de Conservación de Áreas Naturales y de Vida Silvestre, Ley de Hidrocarburos y su reglamento ambiental, Ley de Minería, que incluye aspectos relativos a la preservación del ambiente, Ley de Gestión Ambiental, la cual se refiere al desarrollo sostenible. Asimismo, se crearon derechos de la naturaleza y propuestas que se enfatizan en el Sumak Kawsay o "el Buen Vivir" cuyos objetivos son mantener una relación armoniosa entre los ejes social, político y ambiental que conforman la sostenibilidad, asegurando que las presentes y futuras generaciones puedan satisfacer sus necesidades.

Según Anzil (2006) los recursos naturales son parte de la naturaleza, por ende da lugar a cualquier explotación o aprovechamiento por parte del hombre para de una u otra manera poder satisfacer sus necesidades. Estos recursos pueden clasificarse en renovables y no renovables. Un recurso renovable no se agota, ya que la naturaleza se encarga de regenerarlo con rapidez (Pérez \& Gardey, 2015). El Ecuador es un país con una gran cantidad de recursos naturales, también es conocido por poseer la mayor cantidad de ríos por km2 en el mundo; por tal motivo el uso de estos recursos deben ser utilizados con un alto grado de responsabilidad por parte de las comunidades locales y entidades competentes, para que de esta manera se facilite un mejor desarrollo local y sostenible. Targhetta (2014) aclara que los recursos naturales cumplen un papel fundamental en el desarrollo de la economía mundial, pero que es importante la innovación en el aprovechamiento y uso de estos recursos cuya finalidad sea su conservación. Los recursos naturales en el Ecuador son una fuente muy importante para el desarrollo turístico local e internacional, actualmente el turismo ocupa la tercera plaza generadora de divisas, detrás de la producción de banano y petróleo.

El concepto de desarrollo toma una dimensión mayor en el debate académico y político en el siglo XX con la necesidad de incluir al debate la distribución del ingreso y la inclusión social. Pero no es sólo en términos de desarrollo económico que se orienta el discurso, también es una época en la que se amplían los movimientos en provecho de un desarrollo de menor explotación de la naturaleza. Entonces, nace el desarrollo acompañado de una serie de objetivos, como el desarrollo sustentable (Moreno de Souza et al. 2015). Esto incrementa la posibilidad de mejorar la calidad de vida por parte de las personas que habitan en determinado lugar. La actividad turística no escapa a efectos indeseados y previsibles del impacto de las prácticas inadecuadas e impropias, producto de su espontánea expansión y su incontrolado crecimiento, en perjuicio de su integral desarrollo (Mantero, 2013). Como se ha evidenciado, el turismo se ha transformado en un generador de divisas muy importante para una nación, convirtiéndose a su vez en una amenaza ambiental debido a la gran afluencia de turistas, lo cual como motivo de sus actividades diarias provocan una gran cantidad de residuos y desechos, lo cual conlleva a un impacto ambiental no solo para el lugar, sino para el planeta. Monge \& Yague (2016) señalan la gran importancia del desarrollo del turismo en el Ecuador como parte de una nueva alternancia de la matriz productiva, sujeto a un desarrollo sostenible, donde no solo se busca un crecimiento económico sino también una igualdad social y cultural junto a una armonía ambiental.

Actualmente existen muchos interrogantes sin respuestas acerca del buen uso de los recursos naturales que posee un país, para ello se hace hincapié en lo que señala Sánchez (2011): ¿La abundancia de recursos naturales puede constituirse en un factor decisivo para los procesos de crecimiento económico? o por el contrario, ¿el crecimiento económico se encuentra en conflicto con el medio ambiente? Según estas preguntas se enfatiza que el uso de los recursos naturales brindan un beneficio económico para la sociedad, pero que a su vez crean un vínculo de aspecto negativo logrando así un impacto ambiental perjudicial para la naturaleza, lo cual es un factor importante que debe ser tomado en cuenta por entidades capacitadas para realizar un análisis socioeconómico y ambiental, para que se pueda ejecutar un buen uso y valoración de estos recursos que 
les brinda la naturaleza. Tomio \& Ullrich (2015) indican que el turismo es uno de los campos en los cuales se da el uso de recursos naturales con intencionalidades económica y social. Entre las opciones para su práctica se destacan los segmentos que involucran los recursos naturales como el ecoturismo y el turismo de naturaleza, los cuales buscan y utilizan espacios naturales que permiten el desarrollo de actividades de ocio en contacto con la naturaleza. En estos segmentos, los recursos naturales son tratados como una importante "materia prima" que a su vez es transformada en producto de consumo para los turistas. El uso de esos recursos naturales también implica valores sociales además de los económicos. Es una problemática mundial acerca de este tema en diferentes ámbitos, tanto económico como social y sobretodo ambiental, lo cual ha generado el uso indiscriminado de recursos naturales, obteniendo así un aspecto nocivo para la naturaleza, debido al uso de manera retrograda de éstos, se está perdiendo el valor fundamental de la sostenibilidad no se pone en práctica los ejes principales de ésta, lo que está generando actividades turísticas con malos manejos ambientales por parte de los turistas. La importancia de la presencia de recursos naturales en un territorio representa un crecimiento económico con beneficios a la sociedad, con desventaja ambiental, pero relacionándolas entre sí, se puede encontrar una respuesta positiva para lograr obtener un resultado sostenible, el cual es amigable con el ambiente y por ende con la sociedad y sus ingresos, concluyendo que la valoración de éstos debe tener un grado muy alto de responsabilidad, para no afectar al futuro de las generaciones próximas, logrando conseguir que estos recursos se mantengan en un buen estado de conservación. Tomio \& Ullrich (2015) indican que el turismo es una actividad en creciente expansión en todo el mundo, los agentes de turismo y los investigadores académicos po- drían encarar con más profundidad este tema, visto que la poca valoración de los bienes públicos naturales afecta directamente a la mayoría de los principales atractivos turísticos, cuyos atributos están directa o indirectamente ligados a la naturaleza y sus recursos.

\section{METODOLOGÍA}

Este artículo sintetiza las actividades que se realizaron para obtener datos específicos del cantón Chilla para condensary realizar una investigación relevante y verídica con base a los recursos naturales que son utilizados en el cantón Chilla. La metodología que se utilizó en este trabajo fue la observación directa, que es un instrumento para los investigadores que ayuda a conocer y verificar el estado innato de la realidad en la que se encuentra el objeto a investigarse. Se realizó la búsqueda de información bibliográfica en artículos científicos, la realización de encuestas a los pobladores del cantón, técnica que está más relacionada con el marco teórico de este artículo, ya que se caracteriza por brindar un mejor conocimiento acerca de lo que está siendo investigado en este caso los recursos naturales. Con la utilización de estas herramientas para la investigación se logró obtener resultados reales, los cuales fueron interpretados para tener un conocimiento más específico de lo que sucede con esta problemática que vive el cantón, logrando plasmar en cuadros estadísticos y en números reales la información obtenida.

\section{RESULTADOS}

La información recogida sobre la valoración de cada recurso natural de acuerdo a necesidades diarias por parte de la población del cantón Chilla se presenta en la Tabla 1.

Tabla 1. Valoración de cada recurso natural de acuerdo a necesidades diarias del cantón Chilla

\begin{tabular}{lc}
\hline Etiquetas de fila & Frecuencia (\%) \\
\hline Animales domésticos & 12 \\
Animales silvestres & 9 \\
Arroyos o acequias & 13 \\
Lagos o lagunas & 3 \\
Minerales no metálicos & 2 \\
Minerales metálicos & 1 \\
Plantas comestibles & 17 \\
Plantas medicinales & 14 \\
Plantas silvestres & 10 \\
Ríos & 18 \\
Total & 100 \\
\hline
\end{tabular}

Fuente: Datos de la investigación. 
De acuerdo a las encuestas realizadas a los pobladores del cantón Chilla, el $18 \%$ de la comunidad utiliza el recurso agua proveniente de los ríos que hay en la zona para realizar sus actividades diarias. Uno de ellos el río Chillayacu que es utilizado como fuente de agua para el riego de varias zonas de producción agrícola, además de ser utilizada para el consumo humano y para abrevaderos de fauna.

Haciendo hincapié a lo que indican Ramírez \& Yepes (2011) el recurso agua es indispensable y de vital importancia para el ser humano. Esto conlleva a deducir que este recurso es una fuente de vida importante para la comunidad, del cual también se obtienen beneficios. También especifica que el agua debe ser distribuida de manera equitativa para cada sector, porque se trata de un servicio que la comunidad está destinada a recibir, por la cual las autoridades pertinentes deben tener un sistema de distribución de agua potable para que la población reciba este recurso que es muy útil diariamente. El 17\% de habitantes utiliza las plantas comestibles para su alimentación, siendo el maíz, el frejol y la papa los de mayor consumo y los que cuentan con mayor número de hectáreas producidas. De acuerdo a lo que expresan Cilia et al. (2015) las plantas nativas brindan un beneficio positivo para la sociedad, ya que no solo logra incentivar a los pobladores en el ámbito económico, es decir hacer uso de éstos para su comercialización, sino también son útiles para la alimentación. De esta manera se logra identificar cuáles son los recursos más sobresalientes y de mayor importancia para la población del cantón Chilla, generando así un grado de valoración alto de este tipo de recursos para la calidad de vida de la población local y su desarrollo turístico ya que son parte de los hábitos y costumbres locales y los turistas cada vez quieren inmiscuirse más, conocer de cerca la forma de subsistir de los habitantes de los lugares donde se han alojado, convirtiéndose así en un punto a favor para el desarrollo del turismo local. La flora del cantón tiene plantas comestibles como también algunas especies medicinales que los habitantes utilizan diariamente no solo para uso personal, sino también para la venta, entre ellas están Valeriana (Valeriana officinalis), utilizada como un tranquilizante, lo toman las personas que sufren de alteraciones nerviosas y la Chuquiraga jussieui o chuquiragua que sirve como diurético.

El resultado de las encuestas determinaron que el $54 \%$ de las personas catalogan que en el cantón Chilla tienen un buen manejo de los recursos naturales, tales como el recurso agua, representado por los ríos y pequeños afluentes de agua, la flora con su vegetación de bosques naturales y el suelo, los cuales son de vital importancia para el desarrollo y la subsistencia de esta población (Tabla 2). El segundo mayor porcentaje con un $28 \%$ indica que el buen manejo y uso de estos recursos se los realiza de forma regular. Esto da un porcentaje satisfactorio que demuestra que las personas están teniendo un mayor grado de valoración de los recursos y se están apegando a las buenas prácticas ambientales, por lo cual se está cuidando y manejando de forma responsable y consciente los recursos que ahí existen. Muñoz \& Torres (2015) expresan que un manejo adecuado de recursos naturales causa un bajo impacto ambiental ayudando a generar plazas de trabajo para la población local, convirtiéndose así en un punto a favor para el desarrollo turístico local. Según las aclaraciones de García \& Restrepo (2015) la comunidad debe sistematizar un conjunto de buenas prácticas ambientales para lograr cambios positivos con el fin de cuidar, conservar y obtener beneficios de los recursos naturales que será de gran utilidad para las actividades diarias que realizan y también servirá como punto de atracción para los turistas, por consiguiente esto conlleva una mayor demanda turista, generación de recursos económicos y mejor desarrollo turístico. El uso adecuado de los recursos naturales y la alta valoración que le dan las personas hoy en día es un tema de gran relevancia y de consideración para la conservación y el desarrollo de un lugar, ya que permite cuidar el medio ambiente y preservar el ecosistema, contribuyendo en el desarrollo de un determinado lugar de manera sustentable, lo cual no afectaría a las generaciones futuras.

\section{Tabla 2. Manejo de los recursos naturales}

\begin{tabular}{lc}
\hline Etiquetas de fila & Frecuencia (\%) \\
\hline Bueno & 54 \\
Excelente & 2 \\
Malo & 6 \\
No sabe & 10 \\
Regular & 28 \\
Total & 100 \\
\hline
\end{tabular}

Fuente: Datos de la investigación. 
El $20 \%$ de las personas encuestadas respondieron que la actividad que más se realiza para conservar los recursos naturales en su cantón es la reforestación, con esto se prioriza que las actividades que están realizando son con base a la sostenibilidad, ya que con esto brinda un beneficio a la sociedad y al medio ambiente (Tabla 3 ). Fernández et al. (2013) coinciden que la reforestación con especies forestales maderables permite recuperar en poco tiempo el bosque que se ha perdido a causa de la intervención del hombre. Según Mahecha (2002) las malas prácticas que se realizan de forma tradicional, como quemas, talas y desmontes, disminuye la cantidad de carbono proveniente del suelo, pero esto se puede solucionar ya que existen estudios que indican que la reforestación de bosques de hasta 600 mil hectáreas, ayuda a conservar carbono durante décadas. Mediante la observación directa en el lugar se determinó que existe gran cantidad de bosques que ha sido talados para beneficios de personas con fines lucrativos, gracias a la intervención del Ministerio de Medio Ambiente y autoridades locales han logrado establecer un acuerdo con las personas dedicadas a la extracción de madera, en el cual deben reforestar la zona que ha sido afectada para lograr un cuidado optimo y eficaz de los bosques que están en este sitio. Molina et al. (2016) señalan que por cuestiones climatológicas algunas veces la recuperación de forma natural de vegetación se torna muy trabada y tardía; es aquí donde es necesaria la intervención del hombre con acciones que impliquen una correcta restauración ecológica.

En segundo lugar, con un porcentaje del $14 \%$, el manejo de desechos sólidos también es realizado con la finalidad de preservar los recursos y el ecosistema, en algunos casos estos desechos son provocados por el propio desarrollo del turismo. Entre una de las actividades de manejo de desechos sólidos está el reciclaje de plástico, vidrio y papel, llevado a cabo por los habitantes, no solo por querer ganar un beneficio económico, sino de contribuir al cuidado del medio ambiente del cantón. Esta actividad es muy bien vista y valorada en el desarrollo turístico, ya que cómo lo indica (Andrés \& Torres, 2010) la basura que queda en los atractivos o zonas de desarrollo turístico, deterioran la calidad del paisaje. De esta manera el correcto manejo de desechos sólidos ayudará a la conservación del área y una buena percepción del turista, pues un ambiente tranquilo y bien cuidado es fácilmente captado por las personas para su posible estadía. Siguiendo con el tercer porcentaje, está la ejecución de campañas de limpieza en zonas urbanas y rurales de la localidad, lo cual se lleva a cabo por medio de mingas de los pobladores. Esto ayuda a mejorar la parte visual del lugar, el mejoramiento de su entorno conlleva a un mejor desarrollo, dado que el Cantón Chilla de por sí tiene unos paisajes naturales magníficos que lo complementa su riqueza cultural.

\section{Tabla 3. Actividades a favor del cuidado y conservación de los recursos naturales}

\begin{tabular}{lc} 
Etiquetas de fila & Frecuencia (\%) \\
\hline Ahorro de agua potable & 7 \\
Campaña limpieza bosques & 7 \\
Campaña limpieza ríos & 8 \\
Campaña limpieza zonas & 11 \\
Manejo de desechos agrícolas & 8 \\
Manejo desechos líquidos & 5 \\
Manejo desechos sólidos & 14 \\
No se han ejecutado & 3 \\
Prevención de incendios forestales & 10 \\
Procesos agrícolas ecológicos & 3 \\
Protección de animales silvestres & 4 \\
Reforestación & 20 \\
Total & 100 \\
\hline
\end{tabular}

Fuente: Datos de la investigación. 
En la Tabla 4 el mayor porcentaje es el reciclaje, con el $26 \%$ de personas encuestadas que han respondido que esta es la capacitación que más han recibido en su comunidad. Como señalan Ruiz et al. (2015), en la actualidad los nuevos cambios en los estilos de vida de la población mundial ha permitido crear un tipo de hábito poco consciente en cuanto a la compra excesiva de productos que en algunos casos son de muy poca utilidad para aquellos que los adquieren, generando así un escenario innecesario de producción de desechos sólidos para el medio ambiente. Por lo tanto estas capacitaciones sobre el reciclaje permiten a las personas conocer y poner en práctica una forma de cuidar el medio ambiente, buscando un desarrollo turístico en la zona, este tipo de ejecuciones será de vital importancia para su buen manejo. El reciclaje forma parte de las tres $\mathrm{R}$ de la ecología, propuesta que está siendo muy utilizada a nivel mundial por grandes empresas para generar un buen ambiente sostenible en el desarrollo de sus actividades. La práctica del reciclaje, de acuerdo a Reyes et al. (2015) es una forma de reutilizar los objetos que ya no se utilizan; esto encaja en las buenas prácticas ambientales que tienen los pobladores de este cantón, porque les permite dar una segunda utilidad a las cosas que ya están deterioradas o que ya no utilizan, esto es un beneficio no solo para la comunidad o el medio ambiente, sino también para el desarrollo turístico del lugar. Como mencionan García et al. (2012) los desechos deben de ser observados no solo como un problema sino más bien como una nueva oportunidad para los pobladores los cuales ayuden a generar recursos o algún beneficio con su aprovechamiento. El mal manejo de desechos ya sean sólidos o líquidos tiene un valor influyente para muchos turistas, ya que pueden llevarse consigo una mala impresión del lugar por problemas de contaminación y a su vez podría influenciar en la toma de decisiones de otros posibles visitantes. Cabe acotar que sin la llegada de turistas al cantón no se podrán generar recursos económicos, plazas de trabajo y por ende no podrá haber un buen desarrollo turístico en la localidad.
El uso de abonos orgánicos para la actividad agrícola ha sido un tema que se ha tratado por parte de las entidades gubernamentales en esta localidad, incluso se ha instalado biodigestores, los cuales ayudan a generar fertilizantes a partir de otros residuos orgánicos como excremento de los propios animales y otros tipos de desechos. Las capacitaciones y charlas han ayudado a conocer los beneficios que brinda la utilización de este tipo de fertilizante, dado que existe cada vez mayor contaminación en los cultivos que son producidos por fertilizantes químicos y corrosivos para la salud humana. De acuerdo a lo que indican Fortis-Hernández et al. (2009) este tipo de fertilizantes han sido utilizados desde épocas remotas, cuya finalidad ha sido la rehabilitación o mejoramiento de suelos en etapa de degradación, consiguiendo así volver a retribuir con nutrientes lo que ya se ha perdido por la utilización de fungicidas, pesticidas y demás materiales químicos que se han manejado en la actividad agrícola.

Las capacitaciones sobre problemas ambientales que existen no sólo en este lugar, sino en todo el mundo, se han venido desarrollando con la finalidad de despertar en las personas conciencia sobre los efectos negativos que causa la contaminación en la salud humana y en el desarrollo del turismo local. Según García (2002) las personas deben ser educadas sobre temas de concienciación y responsabilidad ambiental; esto ayudará al mejoramiento de sus hábitos cotidianos, asegurando una mejor toma de decisiones al momento de querer resolver un problema ambiental.

Por estas razones autoridades y entidades competentes, como el Ministerio de Agricultura, Ganadería, Acuacultura y Pesca (MAGAP); Ministerio de Turismo; y Ministerio del Ambiente, han considerado importante desarrollar proyectos, capacitaciones, charlas a la población con la finalidad de tener conciencia del plan de remediación ambiental que se debe ejecutar a fin de minimizar la degradación de recursos naturales para que no afecte la calidad de vida de las personas residentes y turistas. 
Tabla 4. Capacitaciones realizadas en temas ambientales

\begin{tabular}{lc}
\hline Etiquetas de fila & Frecuencia (\%) \\
\hline Conservación de animales silvestres & 2 \\
Conservación de la flora del lugar & 12 \\
Contaminación del suelo & 9 \\
Elaboración y uso de abono orgánico & 17 \\
No ha recibido capacitación & 2 \\
Problemas ambientales & 14 \\
Producción agrícola ecológica & 7 \\
Reciclaje & 26 \\
Reforestación y cuidado de bosques & 12 \\
Total & 100 \\
\hline
\end{tabular}

Fuente: Datos de la investigación.

Con los resultados de las encuestas se determinó que el $25 \%$ de las personas utilizan como recursos para sus actividades diarias a las semillas para su alimentación, mientras que el segundo bien que se ha extraído de la flora es la medicina natural con un 22\% (Tabla 5). Por lo tanto las semillas toman un valor importante en la gastronomía y calidad de vida de los habitantes y turistas que visitan este lugar. Entre ellas tenemos frijol (Phaseolus vulgaris), maíz (Zea mays), arveja (Pisum sativum), y haba (Vicia faba). En relación a las semillas y otras especies de flora, Sol et al. (2016) indican que la biodiversidad que ofrece la flora de un lugar determinado es un recurso útil para la sociedad que habita en ella. En este caso el resultado es que la mayoría de las personas utilizan las semillas y medicina natural para su alimentación diaria, lo que indica que estos recursos son de vital importancia para la subsistencia de los habitantes de esta localidad y para el desarrollo turístico de la misma; forman parte de la gastronomía del lugar representado en los platos típicos y productos que se ofrecen al turista. La medicina natural y las semillas brindan beneficios a la calidad de vida de la población, tanto las semillas que son una fuente para crear o reproducir nuevas plantaciones de vegetales, árboles que brindan madera, flores, entre otros y las plantas medicinales que son utilizadas para el alivio o sanación de enfermedades o accidentes domésticos.

El $17 \%$ de los encuestados respondieron que utilizan el recurso madera para actividades cotidianas; entre las cuales están laurel (Cordia alliodora), caña guadua (Guadua angustifolia), guayacán (Tabebuia chrysantha), cedro (Cedrela odorata L.), balsa (Ocrhoma pyramidale), y copal (Himenaca courbaril L.).

A nivel mundial la industria maderera es una actividad con fines lucrativos; como lo indica Pinkus (2016), otra ocupación que representó el uso y manejo de recursos naturales por parte de los pobladores fue el corte de madera. Ha sido una actividad constante desde hace varios siglos. Estas acciones realizadas por las personas que habitan un determinado lugar, son utilizadas también para el diario vivir, con estos árboles realizan casas, artesanías y leña; las cuales brindan un beneficio a la población, aunque también tiene un efecto negativo en la sostenibilidad, debido a que genera un impacto ambiental por la tala de árboles, lo cual debe ser atendido por autoridades pertinentes del lugar.

\section{Tabla 5. Valoración de los bienes o servicios que se extrae del recurso flora}

\section{Etiquetas de fila}

Artesanías

Forraje para animales

Frutas

Leña

Madera

Medicina natural

Otros (especifique)

Semillas

Total

\section{Frecuencia (\%)}

Fuente: Datos de la investigación. 
De acuerdo a los datos obtenidos en la Tabla 6, el principal uso que los habitantes hacen de la fauna del lugar es para su propio consumo; es decir, tiene el valor más alto en el desarrollo de su subsistencia, se alimentan de la carne que éstos les brindan. En segundo lugar está el 17\% de la fauna que es utilizado para realizar artesanías propias, también es utilizada como medio de transporte ocupando el $17 \%$ de animales como mulas y caballos, esto se debe a que las personas que habitan en el cantón realizan actividades en el campo utilizando animales de carga. Algunas de las especies de animales más comunes de las que hacen uso para su consumo son las ovejas; por otra parte el ganado vacuno, como vacas; el porcino, conformado por cerdos; aves, como las gallinas; y una especie de roedores, como el cuy. Cabe recalcar que no son animales silvestres, más bien son domésticos criados por los propios habitantes, pero también existe un porcentaje de consumo de animales silvestres producto de la caza, como lo indica la Tabla 6.

Coincidiendo con Barrasa (2012) la población o sociedades han hecho uso de lo que brinda la naturaleza a lo largo del tiempo, esto basándose en su forma y calidad de vida. En la mayoría de casos los habitantes de una comunidad se adueñan de ciertos recursos naturales, aprovechándolos y en algunos casos sobre explotándolos, sin tener conocimientos ambientales que forme relación con la sustentabilidad y el mejoramiento de la calidad de vida, llegando incluso a darle un alto grado de valoración a algunos bienes que se obtienen de ellos, pero que no saben cómo sobrellevarlos para que no exista una degradación ambiental. Tal es el caso del mantenimiento y criadero de ganado para el consumo humano, en donde Alcides \& Cabeza (2010) indican que el ganado vacuno bebe comúnmente de 2 a 7 veces en el día cuando tienen el recurso agua disponible de manera fácil. Esto indica que si los pobladores no saben sobrellevar el manejo y criadero de este tipo de animal estarán afectando de manera directa al hábitat donde este se encuentra, ya que el agua es un recurso natural esencial para la vida de otras especies y su mal manejo puede llevar a su contaminación o agotamiento de la misma.

Con respecto al segundo uso de los bienes o servicio que brinda la fauna del cantón Chilla, tal y como lo muestra la Tabla 6, con mayor valoración está la cacería por parte de la población. Alcides \& Cabeza (2010) expresan que la importancia de la cacería se ve desarrollada desde el punto de vista como una manera normal de subsistencia, el problema aquí se hace grande o de consideración cuando esta práctica ya se la realiza con fines de lucro, rompiendo leyes y normas que pretenden conservar a especies vulnerables para su extinción.

\section{Tabla 6. Uso y valoración de los bienes o servicios que se extrae de la fauna}

\begin{tabular}{lc}
\hline Etiquetas de fila & Frecuencia (\%) \\
\hline Artesanía & 17 \\
Caza para subsistencia & 17 \\
Caza para venta de mascotas & 7 \\
Consumo humano & 31 \\
Cuero & 10 \\
Ninguno & 0 \\
Transporte personas/materiales & 17 \\
Total & 100 \\
\hline
\end{tabular}

Fuente: Datos de la investigación.

Conforme a los resultados obtenidos sobre los recursos naturales del cantón que comercializan los pobladores tenemos los frutos y semillas con el $18 \%$, seguido de la comercialización de carne con el $14 \%$, producido por la ganadería (Tabla 7). La comercialización de bienes o servicios obtenidos del aprovechamiento del capital natural ha sido parte importante y de consideración para el desarro-
Ilo del cantón Chilla, ya que la generación de recursos económicos por este rubro ha servido para la mejora de calidad de vida de la población. La Comisión Nacional para el Conocimiento y Uso de la Biodiversidad (CONABIO, 1998) coincide que para que haya un desarrollo sustentable en un determinado lugar es necesario tener una estabilidad entre la actividad económica y la calidad de vida de una 
comunidad. Tal equilibrio implica tener el conocimiento tanto de los beneficios o aspectos positivos que conllevan la ejecución de actividades económicas como también de los efectos negativos que provoca el aprovechamiento de los recursos naturales que nos brinda determinado lugar.

El aprovechamiento del capital natural, en este caso la comercialización de carne proveniente de la fauna, ya sea doméstica o silvestre, son prácticas desarrolladas comúnmente en zonas rurales; éstas se han venido ejecutando desde épocas pasadas, por comunidades indígenas, las cuales crían ganado y venden su carne en áreas urbanas y rurales. Dicha actividad si no se maneja de forma adecuada puede presentar una amenaza en la conservación de recursos naturales (Méndez \& Montiel (2007). Sin embargo, la creación de programas o proyectos oportunos que busquen la conservación de biodiversidad de especies tanto de flora o fauna, es una buena opción o alternativa para preservar las especies y generar a su vez beneficios a los habitantes de la localidad (Quijano \& Calmé, 2002).

En cuanto al aspecto turístico, la comercialización de estos bienes brinda más opciones a los turistas en relación al servicio de restauración. La diversidad de frutos, semillas y animales para consumo humano permiten tener una gastronomía más variada y exquisita dentro de una localidad. Esto atrae al turista ya que es uno de los aspectos culturales y gastronómicos que todo visitante busca experimentar. Hay que tener en cuenta que la gastronomía es parte de la cultura de una sociedad y trae consigo una serie de ventajas; tales como aumentar el ingreso de recursos económicos a los pobladores, puede ser utilizado o aprovechado como un recurso turístico vinculando a los visitantes con los residentes, ayudando así a producir un desarrollo turístico sustentable (Clemente et al., 2008). Como plantean López \& Sánchez (2012) cada vez más el turista busca experimentar nuevas sensaciones y experiencias, ya no solo quiere ser un observador sino que quiere incluir otros sentidos, como el olfato, gusto y el tacto, y así disfrutar más del desarrollo de actividades características de la vida diaria de habitantes de una localidad, buscando involucrase en actividades diarias de los comuneros o residentes, disfrutar de su gastronomía, conocer costumbres y tradiciones, entre otros aspectos.

Como último ítem está la leña con el 10\% de utilización por los habitantes de esta zona con fines de sustentación del diario vivir, al igual que las frutas y semillas. A nivel nacional la madera es un recurso natural utilizado para construir viviendas ecológicas, las cuales ayudan al buen funcionamiento de la sostenibilidad, también es sustento para los hogares ya que ésta la convierten en leña u objetos para decorar; a pesar

\section{Tabla 7. Comercialización de bienes que se obtienen de los recursos naturales}

\begin{tabular}{lc}
\hline Etiquetas de fila & Frecuencia (\%) \\
\hline Animales silvestres & 3 \\
Artesanías & 4 \\
Carne & 14 \\
Carne de animales & 3 \\
Caza & 1 \\
Cuero & 4 \\
Forraje & 2 \\
Frutos & 18 \\
Leña & 10 \\
Madera & 6 \\
Material pétreo & 3 \\
Medicina natural & 8 \\
Minerales & 0 \\
Ninguno & 0 \\
Peces & 5 \\
Semilla & 18 \\
Total & 100 \\
\hline
\end{tabular}

Fuente: Datos de la investigación. 
Los datos obtenidos indican que las actividades ganaderas se realizan en un $44 \%$, convirtiéndose en la de mayor uso y valoración por parte de los habitantes del cantón en las áreas de bosques, seguido de las actividades agrícolas como su segunda mayor labor (Tabla 8). Este uso podría ser algo negativo en cuanto a la conservación de los recursos naturales. López et al. (2007) coinciden que muchos de los habitantes que tienen terrenos de pastoreo realizan actividades de alto riesgo para los bosques como son manejo de químicos, tala de árboles y quema de flora. Muchas comunidades, sociedades o parte de una población transforman ciertos recursos naturales para obtener beneficios; esto se lo realiza de una forma consciente pero no muy positiva para el medio ambiente, es decir, las personas lo hacen de forma voluntaria, tal es el caso que millones de hectáreas de bosques han sido convertidos en lugares para pastorear el ganado y para la agricultura (Balvanera, 2012). López et al. (2001) también coinciden que muchas veces la falta de comida que sirve de alimento para el ganado (forraje); en estas localidades a veces es escaso, resultado que se obtiene de la fuerte tala de árboles o transformaciones que sufren los bosques ocasionando cambios climáticos un poco drásticos. Sin embargo, esto se puede mejorar, cuidando y preservando la flora del sitio, mejorando las áreas donde se pastorea el ganado con siembra de nuevas especies que sirvan de forraje para los animales. Según el Plan de Desarrollo y Ordenamiento
Territorial del cantón Chilla (2015), la población del cantón está vinculada directamente a la producción agrícola y ganadera en su mayoría. Como toda población rural, su alimentación depende en forma importante y representativa de las cosechas y cría de animales que se puedan obtener en su territorio y se complementa con la venta de ciertos excedentes productivos y adquisición de alimentos provenientes de fuera de la finca. Guzmán-González et al. (2005) destacan que las familias rurales se incorporan cada vez más a actividades distintas de la agricultura, porque representan un apoyo importante para compensar los bajos ingresos agrícolas. Porque con estas acciones generan un sustento nuevo de ingreso, lo cual es un beneficio para las personas que habitan en un lugar del cual viven a base de actividades agrícolas. Como ya se ha mencionado esto es lo que ocurre en este cantón de la provincia de EI Oro, las comunidades están inmersas a estas actividades para subsistir.

El turismo incide de gran manera, ya que la belleza paisajistica y el cuidado de recursos naturales son el atractivo para muchas personas, en este caso los turistas. Si no se maneja de manera adecuada los recursos naturales, como los bosques, perderán valor frente a los ojos del turista. Frente a la pregunta, ¿qué más se podría observar y disfrutar de un lugar que solo tenga amplios terrenos poblados de ganado?, la respuesta es... jla verdad es que muy poco!

\section{Tabla 8. Actividades que se realizan en las áreas de bosque}

\begin{tabular}{lc}
\hline Etiquetas de fila & Frecuencia (\%) \\
\hline Agrícolas & 33 \\
Extracción de madera & 22 \\
Ganaderas & 44 \\
Otros (especifique) & 0 \\
Total & 100 \\
\hline
\end{tabular}

Fuente: Datos de la investigación.

De acuerdo a los encuestados con un total del $49 \%$, se encuentran preocupados por los problemas que afectan el aprovechamiento de los recursos naturales, entre ellos están la pérdida de animales y su hábitat natural, disminución de bosques, incendios forestales, y contaminación del agua (Tabla 9). Esto denota la gran importancia que tienen estos recursos para su subsistencia, pues sin el correcto manejo y uso de los mismos, están conduciendo su desarrollo hacia el desastre, afectando no solo a su vida diaria y personal, sino también al desarrollo turístico del lugar incidiendo en la toma de decisiones de los visitantes, pues un sitio con degradación ambiental no es bien visto por nadie.

El desarrollo del turismo local cada día toma mayor relación entre el hombre y los recursos naturales, lo cual conlleva a desarrollar nuevas formas de mantener un balance en el uso, el cuidado y preservación de recursos, en 
Ios cuales los entes competentes siempre deberán tener participación ya que son los encargados de regular toda actividad que tenga que ver con el uso y manejo de los mismos (Rudzewicz \& Lanzer, 2008). El desarrollo de la actividad turística producida en espacios determinados que posean un ambiente sensible cada vez toma mayor relevancia de estudio por parte de organismos científicos y población mundial (González \& Pelafox, 2006). Se ha venido desarrollando con notoriedad la búsqueda de tácticas que permitan la conservación de especies de fauna, ya que la fauna silvestre tiene un alto valor en la mayoría de turistas en cuanto a la belleza paisajística, trayendo consigo beneficios para los residentes del lugar (Ulloa, 2012). De acuerdo a Quevans et al. (2013), una de las principales causas de pérdida de fauna en el planeta es el tráfico ilegal de animales; algunos son cazados para ponerlos a la venta, siendo América del Sur uno de los lugares con más tráfico de animales. Esto conllevaría a que los turistas se sientan poco atraídos al momento de querer visitar un lugar, ya que la biodiversidad tanto de flora como de fauna provoca en el visitante un deseo de querer conocer ese destino y con la pérdida de especies de fauna se limitaría al desarrollo turístico del lugar.

Otro de los problemas que afecta el aprovechamiento de los recursos naturales son los incendios forestales. Castillo et al. (2003) coinciden que estos incendios han sido desde siempre una forma constante de transformar los paisajes, algunos son producidos de manera natural y otros por la intervención de la mano del hombre. Este tipo de incendios han estado y estarán siempre presentes en todos los ecosistemas existentes, siendo las actividades agropecuarias unas de las principales causantes de los mismos; lamentablemente los daños pueden ser incuantificables y este es uno de los problemas que más temen los habitantes del cantón Chilla ya que no sólo afecta a cierto grupo de habitantes, sino a todo el ecosistema y al desarrollo del turismo.

El agua es otro de los recursos que tiene problemas como la contaminación de vertientes y ríos. Gómez et al. (2001) indican que en la actualidad la contaminación de los recursos hídricos recursos se ha convertido en problema grave y de consideración, la contaminación de ríos u otras fuentes de agua son producidos por desechos urbanos, la actividad agropecuaria, minera, representando un gran peligro para el medio ambiente y por ende para las personas. Los residuos domésticos poseen una gran cantidad de microrganismos de origen fecal, éstos a su vez son vertidos a ríos, arroyos, lagos y otras fuentes de agua (Arcos et al., 2005). El manejo de desechos o residuos sólidos es un problema serio de contaminación, dentro de los que conforman los desechos sólidos están la materia orgánica, plástico, vidrio, y papel (Silva \& De Conto, 2008).

Tabla 9. Importancia de los problemas que afectan y limitan el aprovechamiento de los recursos naturales

\begin{tabular}{lc}
\hline Etiquetas de fila & Frecuencia (\%) \\
\hline Muy preocupado & 1 \\
Nada preocupado & 29 \\
No sabe & 5 \\
Poco preocupado & 16 \\
Preocupado & 49 \\
Total & 100 \\
\hline
\end{tabular}

Fuente: Datos de la investigación.

\section{CONCLUSIONES}

Una de las fuentes de ingresos económicos de una población es el desarrollo del turismo local, el mismo que debe ser realizado considerando la vulnerabilidad de los ecosistemas y respetando y cuidando el entorno natural. El cantón Chilla tiene una gran afluencia de turistas cada año por lo que es necesario concienciar a la población en el cuidado y buen manejo de los recursos naturales que poseen.

El cantón posee una gran riqueza religiosa conocida nacional e internacionalmente, ya que en su territorio se encuentra la Iglesia en donde se venera cada año en el mes de septiembre a la Virgen de la Natividad de Chilla, hecho 
que congrega a miles de feligreses y turistas nacionales e internacionales, que además de realizar turismo religioso, recorren la ciudad, sus parroquias, campos, hermosas lagunas de Cuicocha, disfrutan del bello paisaje que ofrece este cantón de la parte alta de la Provincia de El Oro; degustando de la exquisita gastronomía, como el exquisito caldo de borrego, auténtico y típico plato de Chilla, no se queda atrás el caldo de gallina criolla, y uno de los platos que son atractivo para los turistas es el cuy asado en brazas, animales que son criados por los pobladores del cantón lo que de da un valor agregado a su variada gastronomía.

Sus bellos paisajes, su singular flora entre ellas las bellas orquídeas incrustadas en los grandes árboles que embellecen todo el camino de ida al cantón, razones suficientes para que el Ministerio de Turismo, el Gobierno Autónomo Municipal del Cantón Chilla, los Gobiernos Parroquiales y la población realicen propuestas para elaborar proyectos encaminados al buen uso de los recursos naturales con buenas prácticas ambientales. De esta manera se fomentará el desarrollo de un turismo sustentable que permitirá una mayor afluencia de turistas e ingresos económicos para el cantón, creando una ventaja competitiva frente a otros lugares turísticos con iguales características que Chilla.

\section{REFERENCIAS}

Alcides, S., Cabeza, K. (2010). Importancia de la conducta animal para el manejo productivo de la fauna silvestre y doméstica. Revista Colombiana de Ciencia Animal 2, 1 , 175-214.

Andrés, J.M., Torre, M.G. (2010). Las buenas prácticas ambientales en turismo. Anuario de Estudios en Turismo 6, 46-59.

Anzil, F. (2006). Zona Económica. Recuperado el 4 de diciembre de 2016. Disponible en http://www.zonaeconomica.com/definicion/recursos-naturales

Arcos, M.P., Ávila de Navia, S.L., Estupiñán, S.M., Gómez, A.C. (2005). Indicadores microbiológicos de contaminación de las fuentes de agua. Publicación Científica en Ciencias Biomédicas 3, 4, 69-79.

Balvanera, P. (2012). Los servicios ecosistémicos que ofrecen los bosques tropicales. ECOSISTEMAS Revista Científica y Técnica de Ecología y Medio Ambiente 21, 1 , 136-147.

Barrasa, S. (2012). Conocimiento y usos tradicionales de la fauna en dos comunidades campesinas de la Reserva de
Biosfera de la Encrucijada, Chiapas. Revista Etniobiología 10, 1, 16-28.

Bertoni, M. (2005). Recursos naturales en nodos turísticos. Aportes y Transferencias 9, 2, 95-111.

Castillo, M., Pedernera, P., Peña, E. (2003). Incendios forestales y medio ambiente: Una síntesis global. Revista Ambiente y Desarrollo 19, 3, 44-53.

Plan de Desarrollo y Ordenamiento Territorial del Cantón Chilla. (2015). Recuperado el 22 de agosto de 2017. Disponible en http://www.chilla.gob.ec/index.php/ gad-municipal

Cilia, V.G., Aradillas, C., Díaz-Barriga, F. (2015). Las plantas comestibles de una comunidad indígena de la Huasteca Potosina, San Luis Potosí. Entreciencias Diálogos en la Sociedad del Conocimiento 3, 7, 143-152.

Clemente, J.S., Roig, B., Valencia, S., Rabadán, M.T., Martínez, C. (2008). Acitud hacia la gastronomía local de los turistas: Dimensiones y segmentación del mercado. PASOS Revista de Turismo y Patrimonio Cultural 6, 2, 189 198.

CONABIO. Comisión Nacional para el Conocimiento y Uso de la Biodiversidad. (1998). La Diversidad Biológica de México. Estudio de País. México D.F. 341p.

Delgado, A.T. (2013). Turismo y sostenibilidad. Una propuesta metodológica para el estudio de la sostenibilidad turística a escala municipal. Investigaciones Turísticas 5, 163-171.

Fernández, L., Ramírez, N., González, M. (2013). Reforestación con Cupressus lusitanica y su influencia en la diversidad del bosque de pino-encino en Los Altos de Chiapas, México. Botanical Sciences 91, 2, 207-216.

Ferrán, Y., García, L., Naranjo, M.M., Marrero, M. (2016). La marca país (2010-2014): Ecuador ama la vida y su incidencia en la matriz productiva y el plan del buen vivir. UNIANDES EPISTEME Revista de Ciencia, Tecnología e Innovación 3, 2, 103-112.

Fortis-Hernández, M., Leos-Rodríguez, J.A., Preciado Rangel, P., Orona-Castillo, I., García Salazar, J.A., García-Hernández, J.L., Orozco-Vidal, J.A. (2009). Aplicación de abonos orgánicos en la producción de maíz forrajero con riego por goteo. Terra Latinoamericana 27, 4 , 329-336.

García, L.E., Restrepo, A.C. (2015). Desarrollo humano y social en las prácticas ambientales de los graduados de la maestría en desarrollo sostenible y medio ambiente, Universidad de Manizales. Revista Virtual Universidad Católica del Norte 44, 253-266.

García, J.E. (2002). Los problemas de la educación ambiental: ¿Es posible una educación ambiental integradora? Revista Investigación en la Escuela 8, 46, 5-25. 
García, J., Paz, A., Hernández, P. (2012). Gestión del reciclaje de residuos sólidos desde un enfoque racional. Revista Multiciencias 12, 39-44.

Gómez, L., Larduet, Y., Abrahantes, N. (2001). Contaminación y biodiversidad en ecosistemas acuáticos. El fitoplacton de la Bahía de Santiago de Cuba. Revista de Investigaciones Marinas 22, 3, 191-197.

Guzmán-González, I., Santiago-Cruz, M.J., Leos-Rodríguez, J.A., González-López, G., Martínez-Damián, M.Á., Salas-González, J.M. (2005). El papel del empleo rural no agrícola en las comunidades rurales: Un estudio de caso en Tepeji del Río, Hidalgo, México. Agricultura, Sociedad y Desarrollo 2, 2, 51-63.

González, M., Pelafox, A. (2006). Producción del espacio turístico en ambientes sensibles. Estudios y Perspectivas en Turismo 15, 2, 149-178.

López, M., Jiménez, G., De Jong, B., Ochoa, S., Nahed, J. (2001). El sistema ganadero de montaña en la región norte-tzotzil de Chiapas, México. Veterinaria México 32, 2 , 93-102.

López, T., Sánchez, S.M. (2012). La gastronomía como motivación para viajar. Un estudio sobre el turismo culinario en Córdoba. PASOS Revista de Turismo y Patrimonio Cultural 10, 5, 575-584.

López, F., Gómez, R., Harvey, C., López, M., Sinclair, F. (2007). Toma de decisiones de productores ganaderos sobre el manejo de los árboles en potreros en Matiguás, Nicaragua. Revista Agroforestería de las Américas 45, $93-$ 100.

Mahecha, L. (2002). El silvopastoreo: Una alternativa de producción que disminuye el impacto ambiental de la ganadería bovina. Revista Colombiana de Ciencias Pecuarias 15, 2, 226-231.

Mantero, J.C. (2013). Desarrollo turístico: De la ética de los principios. PASOS Revista de Turismo y Patrimonio Cultural 11, 1, 217-227.

Martin, I., Martín, F. (2013). Reflexiones sobre el concepto de turismo sostenible y su integración en el actual contexto sociopolítico ecuatoriano. Revista Amazónica Ciencia y Tecnología 2, 2, 69-88.

Méndez, F., Montiel, S. (2007). Diagnóstico preliminar de la fauna y flora silvestre utilizada por la población Maya de dos comunidades costeras de Campeche, México. Revista Ecosistemas y Recursos Agropecuarios 23, 2, 127-139.

Molina, J., Ramón-Vallejo, V., Villagrosa, A. (2016). Evaluación de la supervivencia y del crecimiento en la reforestación del proyecto piloto de Albatera tras 12 años. Revista
Científica y Tecnológica 3, 2, 40-49.

Monge, J.G., Yague, J.M. (2016). El desarrollo turístico sostenible. Tren Crucero del Ecuador. Estudios y Perspectivas en Turismo 25, 1, 57-72.

Moreno de Souza, J.W., Narciso, S.F., Rosado, P.A. (2015). Desarrollo sustentable. Un índice para el circuito turístico Trilha dos Inconfidentes - Minas Gerais, Brasil. Estudios y Perspectivas en Turismo 24, 3, 547-569.

Muñoz, E., Torres, G. (2015). Potencial turístico de la microcuenca del rio Chimborazo, cantón Riobamba, Provincia de Chimborazo, Ecuador. European Scientific Journal 11, 23, 325-342.

OMT. Organización Mundial de Turismo. (2013). Recuperado el 4 de diciembre de 2016. Disponible en http:// www2.unwto.org/es/search/node/definicion\%20de\%20 turismo

Pérez, J., Gardey, A. (2015). Definición de recursos renovables. Recuperado el 4 de diciembre de 2016. Disponible en http://definicion.de/recursos-renovables/

Pinkus, M.Á. (2016). Dinámica en el uso de los recursos naturales en el oriente de Yucatán durante el Siglo XX. Pueblos y Fronteras Digital 11, 21, 92-113.

Quevans, N., Falcón, N., Elías, R. (2013). Fauna silvestre y productos derivados decomisados durante el período 2000-2007, Lima, Perú. Revista Salud y Tecnología Veterinaria 1, 1, 14-18.

Quijano, E., Calmé, S. (2002). Patrones de cacería y conservación de la fauna silvestre en una comunidad Maya de Quintana Roo, México. Revista Etnobiología 2, 1, 1-18.

Quispe, D.J., Sánchez, G. (2011). Encuestas y entrevistas en investigación científica. Revista de Actualización Clínica 10, 490-494.

Quizhpe, W., Veintimilla, D., Aguirre, Z., Jaramillo, N., Pacheco, E., Vanegas, R., Jadán, O. (2017). Unidades de paisaje y comunidades vegetales en el área de Inkapirca, Saraguro, Loja, Ecuador. Bosques Latitud Cero 7, 1, 102122.

Ramírez, M.F., Yepes, M.J. (2011). Geopolítica de los recursos estratégicos: Conflictos por agua en américa latina. Revista de Relaciones Internacionales, Estrategia y Seguridad 6, 1, 149-165.

Reyes, A., Pellegrini, N., Reyes, R. (2015). El reciclaje como alternativa de manejo de los residuos sólidos en el sector minas de Baruta, Estado Miranda, Venezuela. Revista de Investigación 39, 86, 150-170.

Rojas, I.R. (2011). Elementos para el diseño de técnicas 
de investigación: Una propuesta de definiciones y procedimientos en la investigación científica. Tiempo de Educar 12, 24, 277-297.

Rudzewicz, L., Lanzer, R.M. (2008). Ecoturismo y conservación de los ecosistemas reservas particulares de patrimonio natural en Brasil. Estudios y Perspectivas en Turismo 17, 3, 226-249.

Ruiz, M.E., Luzuriaga, H.A., Rodríguez, Á.C. (2015). El comportamiento del consumidor y la cultura de reciclaje de residuos sólidos: Caso Mancomunidad Patate, Pelileo. Revista Digital del Medio Ambiente 36, 1-15.

Sánchez, M. (2011). ¿Condicionan los recursos naturales el crecimiento económico? Semestre Económico 14, 29, 117-128.

Silva, G., De Conto, S.M. (2008). Manejo de residuos sólidos en un evento turístico. Estudios y Perspectivas de Turismo $17,1,119-139$.
Sol, A., Gómez, E., García, E., Pérez, A. (2016). Flora útil en el Ejido Sinaloa $1^{a}$ sección, Cárdenas, Tabasco, México. Revista Mexicana de Ciencias Agrícolas 14, 2671-2681.

Targhetta, J. (2014). Recursos naturales y desarrollo económico. Recuperado el 12 de febrero de 2017. Disponible en http://www.revista-uno.com/recursos-naturales-y-desarrollo-economico/

Tomio, M., Ullrich, D.R. (2015). Valoración económica ambiental en el turismo. Estudios y Perspectivas de Turismo 24, 1, 172-187.

Ulloa, J. (2012). ¿Por qué debemos conservar la fauna silvestre? Spei Domus 8, 17, 66-69.

Vargas, C. (2016). Importancia de la sostenibilidad ambiental en la ingeniería. Recuperado el 4 de diciembre de 2016. Disponible en https://ingenieriainerva. com/2016/12/04/importancia-de-la-sostenibilidad-ambiental-ingenieria/ 